\title{
TELESSAÚDE: DA IMPLANTAÇÃO AO ENTENDIMENTO COMO TECNOLOGIA SOCIAL
}

\author{
TELEHEALTH: FROM IMPLANTATION TO \\ UNDERSTANDING AS A SOCIAL TECHNOLOGY
}

\author{
Luana Gabriele Nilson ${ }^{1}$ \\ Marcos Aurélio Maeyama \\ Luise Lüdke Dolny ${ }^{3}$ \\ Antonio Fernando Boing ${ }^{4}$ \\ Maria Cristina Marino Calvo ${ }^{5}$
}

RESUMO: O artigo apresenta um resgate histórico da implantação do Telessaúde no âmbito do Sistema Único de Saúde, em especial a experiência do Núcleo de Telessaúde de Santa Catarina. Apresenta os serviços de tele-educação, teleconsultoria, telediagnóstico e segunda opinião formativa como instrumentos de educação permanente a distância para as equipes de Atenção Primária à Saúde, propiciando melhoria da qualidade da assistência e ampliação do acesso à saúde, revelando-se como importante tecnologia social.

PALAVRAS-CHAVE: Telessaúde. Atenção Primária à Saúde. Sistema Único de Saúde. Tecnologia Social.

ABSTRACT: This article presents a historical review of the implementation of Telehealth within the SUS - Brazilian Unified Health System, focusing specifically on the experience of the Santa Catarina Telehealth Center. It presents tele-education, teleconsultation, telediagnostic and formative second opinion as instruments of continuing health education for primary healthcare teams, providing qualification of health care and broadening access to health services, making it an important social technology.

KEYWORDS: Telehealth. Primary Health Care. Unified Health System. Social Technology.

Licença CC BY: Artigo distribuído sob os termos Creative Commons, permite uso e distribuição irrestrita em qualquer meio desde que $o$ autor credite a fonte original.
Enfermeira. Mestre em Saúde Coletiva pela Universidade Federal de Santa Catarina - UFSC. E-mail: luanagnilson@gmail.com.

2 Dentista. Doutor em Saúde Coletiva pela Universidade Federal de Santa CatarinaUFSC. Mestre em Saúde e Gestão do Trabalho pela Universidade do Vale do Itajaí - UNIVALI. Professor titular da UNIVALI. E-mail: marcosmaeyama@ig.com.br.

3 Psicóloga. Mestre em Saúde e Gestão do Trabalho pela Universidade do Vale do Itajaí - UNIVALI. E-mail: luiseludke@gmail.com.

4 Dentista. Doutor em Ciências Odontológicas pela Universidade de São Paulo USP. Mestre em Saúde Coletiva pela Universidade Federal de Santa Catarina UFSC. Professor titular da UFSC.E-mail: antonio.boing@ufsc.br.

5 Dentista. Doutora em Engenharia de Produção e Mestre em Saúde Pública pela Universidade Federal de Santa Catarina - UFSC. Professora titular da UFSC. E-mail: cristina.clv@gmail.com. 


\section{INTRODUÇÃO}

Telessaúde refere-se à prestação de serviços de atenção à saúde por meio do uso de tecnologias de informação e comunicação (TIC), de forma a superar a barreira da distância e aproximar os serviços de saúde; promover acesso e melhorar a qualidade da assistência com apoio à tomada de decisão pelos profissionais; compartilhamento e coordenação de recursos geograficamente distribuídos, otimizando seu uso e garantindo a oferta qualificada de diferentes especialidades em saúde (ORGANIZAÇÃO PANAMERICANA DE LA SALUD, 2016).

A história da telessaúde iniciou com a telemedicina, uma atividade inicialmente médica (ASCENCIO, 2012). Os termos têm o mesmo princípio, mas a telessaúde amplia o conceito para as diversas áreas da saúde e suas ações de promoção e proteção, educação para a saúde, saúde pública e de comunidade (SILVA, 2014).

O alcance da telessaúde tem ampliado à medida que cresce o interesse no seu uso e se estabelecem legislações para orientar as práticas, respaldadas pela busca de mudanças nas perspectivas da assistência em saúde para o futuro, oferecendo mecanismos de apoio e comunicação entre trabalhadores de primeira linha de cuidado (BILL; CRISCI; CANET, 2014).

No mundo todo, as aplicações da telessaúde têm sido por meio de serviços de (ORGANIZAÇÃO PANAMERICANA DE SAÚDE, 2016; ORGANIZAÇÃO MUNDIAL DE LA SALUD, 2016; CÁCERES MÉNDEZ et al., 2011):

- Assistência remota: que pode ser direta ao paciente ou por meio de apoio ao profissional, para diagnóstico e tratamento a distância de pacientes, acompanhamento para seguimento ou cuidado e manejo de doentes;

- Gestão administrativa de pacientes: solicitações de provas analíticas e questões relacionadas a cobranças e prestação de serviços;

- Formação e educação a distância em saúde e na saúde: por meio da discussão de temas de interesse com base em evidências para promoção de educação de forma contínua dos profissionais de saúde e também dos usuários em geral dos sistemas de saúde;

- Avaliação e investigação colaborativa em rede: compartilhamento das melhores práticas e construção de conhecimento por meio de referência e contrarreferência entre os envolvidos.

Com a utilização da telessaúde, cinco benefícios fundamentais podem ser alcançados: 1) melhoria do acesso à saúde e à qualificação profissional; 2) eficiência - reduzindo custos nos cuidados, na formação profissional, com deslocamento e tratamento institucional de pacientes; 3) melhoria da qualidade e da resolubilidade dos serviços de saúde; 4) redução do tempo de espera por serviços necessários; 5) satisfação dos pacientes, famílias e comunidade em utilizar telessaúde, pela melhoria do acesso e aproximação dos serviços que precisam (ORGANIZAÇÃO PANAMERICANA DE LA SALUD, 2016; AMERICAN TELEMEDICINE ASSOCIATION, 2016; CÁCERES MÉNDEZ et al., 2011). A telessaúde promove assim a orientação do modelo de atenção à saúde para a centralidade nos usuários, com globalidade e interoperabilidade entre as organizações de saúde para o trabalho em rede (ORGANIZAÇÃO PANAMERICANA DE LA SALUD, 2016).

Este artigo se propõe a apresentar a telessaúde a partir de um resgate da história do Programa Nacional Telessaúde Brasil Redes e da apresentação do Núcleo Telessaúde Santa 
Catarina. Contempla uma revisão teórica e o relato acerca da estruturação e da oferta de serviços de telessaúde em Santa Catarina, discutindo a telessaúde como tecnologia social.

\section{A TELESSAÚDE NO BRASIL: HISTÓRIA E IMPORTÂNCIA ESTRATÉGICA PARA O FORTALECIMENTO DO SUS}

A necessidade crescente de atenção às demandas em saúde fez emergir um contexto favorável ao surgimento da telessaúde como forma de descentralizar os serviços de saúde e ampliar o acesso dos usuários; agilizar e qualificar os atendimentos, por meio do incentivo de maior permanência possível dos usuários junto às suas equipes de saúde de referência. Isso se tornou mais viável a partir da criação do Sistema Único de Saúde (SUS) em 1990, em um momento em que o Movimento Brasileiro de Reforma Sanitária ganhou forças, e com ele a compreensão de que o foco da atenção precisa ser sempre o usuário, o que requer mais do que alta densidade tecnológica para seu cuidado (BRASIL, 1988; BRASIL, 1990).

Contudo, mesmo com a criação do SUS e a consagração de seus princípios - universalidade, equidade e integralidade -, permaneceu a desigualdade de acesso a serviços de saúde, especialmente em municípios distantes dos grandes centros. Nesses locais, geralmente, os médicos que atuam na Atenção Primária à Saúde (APS) são jovens e têm pouca experiência profissional (MARCOLINO et al., 2013), sentem-se isolados e há grande rotatividade profissional (PAIM et al., 2011).

Para apoiar esses profissionais, a telessaúde é uma das estratégias do Ministério da Saúde (MS), promovendo serviços e assistência à saúde de qualidade por meio de Educação Permanente em Saúde (EPS) - regulamentada pela Política Nacional de EPS (PNEPS) (BRASIL, 2009), para transformação de práticas e alcance das competências necessárias ao modelo de atenção que tem a APS como coordenadora do cuidado e ordenadora da atenção.

A história da telessaúde no Brasil começou ainda na década de 80, com relatos mais estruturados na década de 90, nas áreas pública e privada, mais centralizados em São Paulo e no Rio de Janeiro, com ações de telecardiologia, de videoconferências e o aparecimento dos primeiros sites oficiais sobre saúde (EL-KHOURI, 2003; WEN, 2008). No final da década de 90 e início dos anos 2000, as ações de telemedicina se difundiram mais no Brasil, nos estados de Santa Catarina, Pernambuco, Paraná, Rondônia, Minas Gerais, Rio Grande do Sul (EL-KHOURI, 2003; WEN, 2013; VON WANGEHEIM; CAVALCANTE; WAGNER, 2013; ALKMIM et al., 2013; MONTEIRO et al., 2013).

O emprego de tecnologias em saúde se expandiu e se fortaleceu na primeira década dos anos 2000 e foi nesta época que o MS iniciou o apoio à telemedicina e à telessaúde com ações pontuais e fomento a projetos gerais, sem sistematização das ações, que atendiam a diferentes finalidades, de acordo com as instituições que as desenvolviam ou coordenavam (EL-KHOURI, 2003).

O ano de 2005 é marcante para a telessaúde no Brasil (SILVA, 2014). Neste ano, as ações de telessaúde voltadas para a APS começaram a se concretizar, por meio do esforço do Departamento de Gestão da Educação na Saúde da Secretaria de Gestão do Trabalho e da Educação na Saúde (SGTES) do MS (TELESSAÚDE BRASIL REDES, 2016). Desde o início buscou-se integrar diferentes Ministérios, gestores estaduais e municipais, unindo a expertise da academia com as necessidades em saúde pública, em busca de ampliar o acesso e o uso aos serviços de telemedicina 
e telessaúde, buscando melhorar a qualidade da APS no SUS por meio de TIC para promover teleassistência e tele-educação (TELESSAÚDE BRASIL REDES, 2016).

A Portaria $n^{\circ} 35$ GM/MS, de 04 de janeiro de 2007, instituiu o Projeto Piloto Nacional de Telessaúde Aplicada à Atenção Primária, denominado Programa Nacional de Telessaúde (TELESSAÚDE BRASIL REDES, 2016; SILVA, 2013; BRASIL, 2007), para apoio às ações de educação e assistência à saúde (OLIVIERA et al., 2015; BRASIL, 2012). O Programa do MS foi então criado pela SGTES, visando ampliar a oferta de EPS com foco na APS, por meio da oferta de ações de tele-educação, segunda opinião e telediagnóstico (TELESSAÚDE BRASIL REDES, 2016).

No contexto do Programa, telessaúde é entendida como “o uso das modernas tecnologias de informação e comunicação para atividades à distância relacionadas à saúde em seus diversos níveis (primário, secundário e terciário)" (BRASIL, 2012, p.15). Um Núcleo ou serviço de Telessaúde se caracteriza pelo apoio diagnóstico e terapêutico e o foco no caráter educativo de suas ações em busca da qualificação das ações assistenciais para ampliação da capacidade de resposta às necessidades em saúde da população (BRASIL, 2012).

Os nove estados participantes do projeto piloto foram: Amazonas, Ceará, Pernambuco, Goiás, Minas Gerais, Rio de Janeiro, São Paulo, Santa Catarina e Rio Grande do Sul, por meio de universidades federais e estaduais. Foram implantados pontos de acesso em 100 municípios de cada estado, para assistir aos profissionais da APS de mais de 2.500 equipes, com a distribuição de equipamentos de informática para as equipes e os municípios contemplados, ficando os municípios responsáveis por garantir a conectividade (TELESSAÚDE BRASIL REDES, 2016). Os municípios foram selecionados a partir de critérios de elegibilidade, que incluíam o comprometimento da gestão, baixo IDH e barreiras geográficas de acesso a serviços de referência (BRASIL, 2007).

Um ano após a implantação do Programa Nacional de Telessaúde, a diminuição de deslocamentos de pacientes já havia possibilitado a economia de 35 milhões de reais para o SUS (NASSIF, 2014) e em 2009 a abrangência dos Núcleos de Telessaúde chegava aos 900 municípios, beneficiando 11.000.000 habitantes, e mais 4 estados iniciavam suas atividades (TELESSAÚDE BRASIL REDES, 2016).

Em 2011, a Portaria GM/MS nº 2.546 redefiniu e ampliou o Programa Telessaúde Brasil, que passou a ser denominado Programa Nacional Telessaúde Brasil Redes (Telessaúde Brasil Redes), com o objetivo de apoiar a consolidação das Redes de Atenção à Saúde (RAS), tendo a Atenção Primária como ordenadora no âmbito do SUS (SIMÕES et al., 2013; SILVA, 2013; BRASIL, 2011a). A partir de então, é redefinida a oferta de serviços, que passa a ser composta por: teleconsultorias, telediagnóstico, tele-educação e segunda opinião formativa para promover a educação permanente e o apoio assistencial em saúde (BRASIL, 2012).

Também em 2011 houve a expansão do financiamento para núcleos municipais e estaduais e a integração do Programa Nacional Telessaúde Brasil Redes ao Programa de Requalificação de Unidades Básicas de Saúde, com incentivo à informatização (BRASIL, 2015a; BRASIL, 2011 b).

Os serviços de telessaúde são organizados a partir de Núcleos Técnico-Científicos de Telessaúde (NTCT) - responsáveis por formular e gerir os serviços oferecidos -, e Pontos de Telessaúde - serviços de saúde onde estão os profissionais que usam os serviços - (NILSON et al., 2017). Por meio desses núcleos, os profissionais podem acessar cursos, webpalestras, apoio assistencial e para organização do processo de trabalho através de telefone, mensagem de texto ou conteúdo publicado em portais criados para tal, que exigem infraestrutura e conectividade com acesso dos profissionais a equipamentos telefônicos e de informática (PINTO et al., 2014; NILSON et al., 2017). 
O Telessaúde Brasil Redes é uma rede de serviços que funciona em parceria a partir de diferentes núcleos, que se estruturam em projetos estaduais, regionais ou intermunicipais, por meio do trabalho compartilhado de secretarias estaduais e municipais de saúde, instituições de ensino e serviços de saúde, oferecendo apoio permanente aos profissionais em diferentes serviços e distintos locais (PINTO et al., 2014; BRASIL, 2011b).

Em 2016 existiam mais de 40 núcleos implantados em 23 estados brasileiros e outros em fase de implantação, tendo o telessaúde no Brasil um importante papel no apoio às estratégias para fortalecer o modelo de organização proposto para o SUS e qualificar o cuidado prestado aos usuários, como o PROVAB (Programa de Valorização da Atenção Primária) e o Projeto Mais Médicos para o Brasil (BRASIL, 2016).

O financiamento dos NTCT é público e se dá por meio de repasses periódicos da União para Universidades ou Fundos de Saúde, que promovem a gestão dos recursos de acordo com as orientações legais de cada instituição. A periodicidade e o volume de repasse são definidos em projetos bienais, submetidos ao Fundo Nacional de Saúde, que tem por regra analisar separadamente projetos de custeio e projetos de capital. As instituições que abrigam o NTCT fornecem a estrutura física e sua manutenção. Tais projetos são analisados pela área técnica no Ministério da Saúde, e os valores aprovados estão vinculados à capacidade de oferta dos serviços e à demanda imediata por ampliação dos serviços (BRASIL, 2015a). Além desse financiamento direto do Fundo Nacional de Saúde, Secretarias Estaduais e Municipais de Saúde também podem contribuir com financiamentos parciais ou cedendo carga horária de profissionais para desenvolvimento de serviços de seu interesse, vinculados ao Telessaúde. Em menor escala, projetos de pesquisa financiados pelas agências de fomento também podem contribuir com o aprimoramento de ações e serviços. Mais recentemente, instituições nacionais de referência na prestação de serviços de alta complexidade têm se interessado em apoiar o desenvolvimento de serviços dos NTCT por meio do Programa de Apoio ao Desenvolvimento do SUS (PROAD-SUS).

\section{TELESSAÚDE EM SANTA CATARINA}

A história da telessaúde em Santa Catarina iniciou em 1997, quando foi criado o Grupo Cyclops na Universidade Federal de Santa Catarina (UFSC), que atua desde então com a produção de tecnologias em aplicativos para uso de TIC em saúde (VON WANGEHEIM; CAVALCANTE; WAGNER, 2013). Desde o início das suas atividades, em parceria com a Secretaria de Estado da Saúde da Saúde de Santa Catarina (SES/SC), o grupo pensava e trabalhava para a ampliação do acesso, com menor custo e tempo de espera a laudos de exames e diagnósticos. O trabalho do grupo culminou com a criação da Rede Catarinense de Telemedicina (RCTM), em 2005, inicialmente conectando 5 municípios do extremo oeste catarinense a Florianópolis para a realização de exames via web em larga escala e usando tecnologia inteiramente nacional (VON WANGEHEIM; CAVALCANTE; WAGNER, 2013).

O NTCT do Estado de Santa Catarina (Telessaúde SC) foi um dos núcleos implantados pelo projeto piloto, em 2007, justamente pelo fato de Santa Catarina já ter essa experiência com atividades de telemedicina (VON WANGEHEIM; CAVALCANTE; WAGNER, 2013).

As atividades do Projeto Nacional de Telessaúde Aplicado à APS no Estado de Santa Catarina tiveram início em 2008, contando com a articulação entre o Hospital Universitário e o 
Curso de Pós-graduação em Saúde Pública da UFSC, o Conselho de Secretários Municipais de Saúde (COSEMS) e a Secretaria de Estado da Saúde, incluindo sua Escola de Saúde Pública (VON WANGEHEIM; CAVALCANTE; WAGNER, 2013). Como previsto no projeto nacional, foram distribuídos os computadores em unidades básicas de saúde do interior do estado para a estruturação de uma rede de serviços de educação permanente. Posteriormente, foram entregues mais 470 computadores e 1250 kits multimídia - webcam, microfone e caixas de som - aos municípios, a partir de levantamento de necessidades, adquiridos com recursos de projetos financiados pelo Ministério da Saúde (NÚCLEO TELESSAÚDE SANTA CATARINA, 2017a).

Santa Catarina possui uma plataforma virtual própria para a oferta de serviços de telessaúde mantida por recursos do Telessaúde SC e da SES/SC. Sua implantação ocorreu em 2005 com um projeto piloto na cidade de Quilombo para oferta de telediagnóstico, por meio do serviço de Telemedicina da SES/SC (SAVARIS et al., 2011). Em 2010, houve a integração da plataforma de telediagnóstico com a plataforma de oferta dos outros serviços do Telessaúde, sendo criado o Sistema Catarinense de Telemedicina e Telessaúde (STT), uma plataforma virtual única desenvolvida pelo Grupo Cyclops/UFSC em parceria com a SES/SC e Telessaúde SC, que oferece laudos a distância de diversas modalidades, acesso dos pacientes aos seus exames, webpalestras, teleconsultorias síncronas (em tempo real) e assíncronas (por mensagem de texto), cursos a distância, vídeos e outros materiais de apoio para formação e qualificação profissional (VON WANGEHEIM; CAVALCANTE; WAGNER, 2013; NÚCLEO TELESSAÚDE SANTA CATARINA, 2017a; 2017b).

O alicerce para a estruturação e para a oferta de serviços pelo Telessaúde SC sempre foi a articulação e trabalho intersetorial envolvendo ensino - UFSC -, e serviço - SES/SC -, com orientação pelos fundamentos da EPS para responder às necessidades reais dos profissionais apoiados, buscando transformar e fortalecer a APS e o SUS.

O Telessaúde SC, desde o início de suas atividades, tem ofertado todos os serviços orientados pelo MS, por meio de equipe multidisciplinar com formação em saúde pública e coletiva, saúde da família e especialidades focais condizentes com as necessidades de sua rede. Essa equipe planeja, monitora, avalia, compõe e constrói os produtos que são oferecidos aos profissionais cadastrados (NÚCLEO TELESSAÚDE SANTA CATARINA, 2017a). A equipe é composta por profissionais diretamente vinculados ao Núcleo - celetistas, prestadores de serviço ou bolsistas -, ou aos parceiros - Secretarias de Estado e Municipais de Saúde.

Entre 2008 e 2012, os serviços contemplaram webpalestras, segunda opinião e telediagnóstico. Ainda em 2012 iniciou um processo de reorganização conceitual e de oferta dos serviços, seguindo as novas diretrizes da SGTES - Portaria ${ }^{\circ}$ 2.546/2011 (BRASIL, 201 1a) e do Departamento de Atenção Básica (DAB) - Portaria nº 2.554/2011 (BRASIL, 2011b) e, a partir de 2013, passou-se a utilizar a nomenclatura atual para os serviços oferecidos: teleconsultoria, telediagnóstico, tele-educação e segunda opinião formativa (SOF) (NÚCLEO TELESSAÚDE SANTA CATARINA, 2017a):

Telediagnóstico: Compreende o apoio ao diagnóstico clínico a partir da emissão de laudos de exames (BRASIL, $2011 \mathrm{a}$; BRASIL, 2015b). Em Santa Catarina o serviço foi exclusivamente financiado pela SES/SC até 2017, e é oferecido em parceria com o Telessaúde SC para as áreas de cardiologia e dermatologia. Equipamentos para eletrocardiograma e dermatoscopia foram distribuídos pela SES/SC e Telessaúde SC aos municípios do Estado, que também fazem investimentos e são responsáveis pela manutenção. 
Os exames solicitados pelos médicos da APS são realizados nas unidades de saúde, enviados pelo sistema para a emissão dos laudos por cardiologistas e dermatologistas, sendo depois devolvidos para acesso dos pacientes e dos profissionais responsáveis. Em ambas as modalidades há o treinamento de profissionais dos municípios para a realização dos exames, além da discussão de fluxos e da melhor forma de uso para que o serviço responda às necessidades locais (NÚCLEO TELESSAÚDE SANTA CATARINA, 2017b).

Tele-educação: Inclui a oferta de palestras, conferências, fóruns, aulas e cursos (BRASIL, 2015). As webpalestras são palestras virtuais realizadas semanalmente, ao vivo, abordando temas atuais e da rotina dos profissionais de saúde. Os profissionais interagem com os palestrantes por meio de chat e depois têm acesso ao conteúdo editado que fica disponível no portal do Telessaúde SC. Essa tem sido uma estratégia importante para a educação dos profissionais da APS, inclusive fomentando espaços de aprendizagem coletiva, pois as equipes se reúnem para assistir à apresentação dos temas de seu interesse, discutir com os palestrantes e resolver dúvida dos profissionais, planejando juntos soluções para os problemas locais (NÚCLEO TELESSAÚDE SANTA CATARINA, 2017b).

Os cursos, oferecidos a distância, também têm alcançado um público expressivo. São cursos de curta duração, com certificação, que utilizam a metodologia autoinstrucional, em que o próprio aluno organiza seu tempo de estudo a partir do cronograma proposto. Os temas trabalhados respondem a demandas identificadas junto aos profissionais usuários do serviço e à realidade enfrentada na rede pelo Telessaúde SC, e são desenvolvidos em parceria com a SES/SC (NÚCLEO TELESSAÚDE SANTA CATARINA, $2017 \mathrm{~b}$ ).

São também realizados webseminários, fóruns de discussão e reuniões de matriciamento, que promovem a interação e o compartilhamento de experiência entre os participantes a partir da discussão de temas clínicos ou de processo de trabalho (BRASIL, 2015; NÚCLEO TELESSAÚDE SANTA CATARINA, 2017b).

Teleconsultoria: É uma consulta entre profissionais de saúde, trabalhadores e gestores, e teleconsultores, para esclarecer dúvidas sobre temas clínicos ou de processo de trabalho das equipes, coordenação ou gestão e que pode ser realizada no formato síncrono (agendamento de horário para conversar em tempo real) ou assíncrono (mensagem de texto disponibilizada em até 72h) (BRASIL, $2011 \mathrm{a}$; BRASIL, 2015; NÚCLEO TELESSAÚDE SANTA CATARINA, $2017 \mathrm{~b}$ ).

As teleconsultorias clínicas e de processo de trabalho são ofertadas para todos os profissionais cadastrados e podem ser solicitadas, por livre demanda, para discutir casos ou temas clínicos gerais, epidemiológicos, de condutas populacionais, solicitação de material técnico de apoio, temas organizativos de planejamento, monitoramento, avaliação ou execução de ações e serviços no contexto das redes de atenção (NÚCLEO TELESSAÚDE SANTA CATARINA, 2017b).

Além disso, estão incluídas como etapa compulsória em fluxos de acesso ao nível especializado de atenção, permitindo a discussão dos casos em que o médico da APS tem intenção de encaminhar o paciente para o especialista (NÚCLEO TELESSAÚDE SANTA CATARINA, 2017b). As teleconsultorias que integram fluxos entre a APS e a Atenção Especializada se propõe a contribuir com a redução de encaminhamentos desnecessários e diminuir o tempo de espera por consultas com especialistas, fortalecendo a APS, qualificando e ampliando o acesso necessário ao nível especializado (NÚCLEO TELESSAÚDE SANTA CATARINA, 2017b; BRASIL, 2015a). 
Segunda Opinião Formativa: São respostas sistematizadas e construídas com base em revisão bibliográfica, nas melhores e mais atualizadas evidências científicas e clínicas que servem à formação de diferentes interessados - profissionais, estudantes, trabalhadores de saúde (BRASIL, 2011a; BRASIL, 2015b; NÚCLEO TELESSAÚDE SANTA CATARINA, 2017b). Elas são construídas a partir das perguntas mais relevantes e pertinentes que são enviadas pelos profissionais na forma de teleconsultoria, sendo selecionadas por tratarem de temas importantes, orientados pelos princípios e diretrizes do SUS que têm a APS como central, e são revisadas antes do envio para publicação no acervo on-line da Biblioteca Virtual em Saúde (BVS), onde ficam disponíveis para acesso livre por todos os interessados (BRASIL, $2011 \mathrm{a}$; NÚCLEO TELESSAÚDE SANTA CATARINA, 2017b).

Para garantir um conteúdo de qualidade, bem embasado, atual e simples a ser acessado pelos profissionais, o processo de construção e validação das SOF pelo Telessaúde SC é rigoroso e passa pela discussão coletiva e revisão por pares internos antes do envio à BVS, onde será avaliado seu potencial para publicação (NÚCLEO TELESSAÚDE SANTA CATARINA, 2017b).

O Telessaúde SC busca o diálogo permanente com profissionais e gestores para pautar a oferta de serviços em reais necessidades de apoio e nas especificidades da rede de serviços. Aliado à Regulação, o Telessaúde pode ir além do papel educativo às equipes de saúde, agindo em pontos críticos do acesso dos usuários ao sistema de saúde. Teleconsultorias e tele-educação também podem contribuir com a reestruturação do processo de trabalho das equipes de saúde e com a mudança de prática e ressignificação do conhecimento para o alcance de novas práticas e da qualificação do cuidado em saúde (FIGUEIREDO; GUEDES, 2015).

\section{NÚCLEO TELESSAÚDE SANTA CATARINA: EVOLUÇÃO NA OFERTA DE SERVIÇOS}

Em 2012, foram realizadas mais de 880 teleconsultorias de processo de trabalho, a participação nas webconferências foi de mais de 75\% dos municípios cadastrados e se alcançou uma média de 30 perguntas respondidas por mês pela teleconsultoria clínica (NÚCLEO TELESSAÚDE SANTA CATARINA, 2017a).

Já em 2013, o Núcleo Telessaúde SC passou a investir em ferramentas e recursos humanos para ampliar a oferta de teleconsultorias assíncronas e síncronas, com cobertura de 84\% dos municípios catarinenses (NÚCLEO TELESSAÚDE SANTA CATARINA, 2017a).

Para expandir o uso e a oferta do serviço de teleconsultorias, e em resposta às demandas do MS e do Telessaúde Brasil Redes, o Telessaúde SC implementou, em 2014, ações sistematizadas para apoio às centrais de regulação no estado e nos municípios, por meio de oficinas e discussão de fluxos e protocolos com gestores, coordenadores e profissionais de saúde, destacando o potencial da telessaúde, em especial das teleconsultorias para o fortalecimento e a qualificação assistencial para a tomada de decisão no âmbito da APS, o que promove resolubilidade e reduz o número de encaminhamentos para os outros níveis de atenção. Essas ações se fortaleceram no ano seguinte com início da oferta de teleconsultorias por especialistas focais (NÚCLEO TELESSAÚDE SANTA CATARINA, 2017a).

Em 2015 foram realizados mais de 210.000 exames de telediagnóstico, 114 webconferências, 27 cursos e mais de 4.400 teleconsultorias, sendo que dessas, 72 foram encaminhadas no formato de SOF para publicação pela Bireme (NÚCLEO TELESSAÚDE SANTA CATARINA, $2017 \mathrm{a}$ ). 
Em 2016, cresceram a oferta e o uso de todos os serviços do Núcleo. O destaque foi para o serviço de teleconsultorias, que ultrapassou a marca de 15 mil solicitações atendidas (NÚCLEO TELESSAÚDE SANTA CATARINA, 2017a).

Até outubro de 2017 o Telessaúde SC contava com 1.574 municípios cadastrados, 1.775 equipes e quase 18 mil profissionais atendidos, sendo que os serviços oferecidos atendem 100\% dos municípios catarinenses (NÚCLEO TELESSAÚDE SANTA CATARINA, 2017a). A Figura 1 traz a evolução da oferta de serviços pelo Telessaúde SC.

Após a implantação do Telessaúde SC houve intensa divulgação dos serviços para gestores e profissionais de forma impressa, por áudio e vídeo, com visitas de campo, envio de e-mails, participação em eventos com oficinas ou apresentação de trabalhos, relatando as experiências e o potencial do apoio. A adesão e a utilização dos serviços estão condicionadas ao interesse individual, de equipe ou em termos de gestão local e de inclusão dos mesmos à rotina de trabalho.

Figura 01: Acesso aos serviços oferecidos pelo Sistema Catarinense de Telemedicina e Telessaúde entre 2012 e outubro de 2017
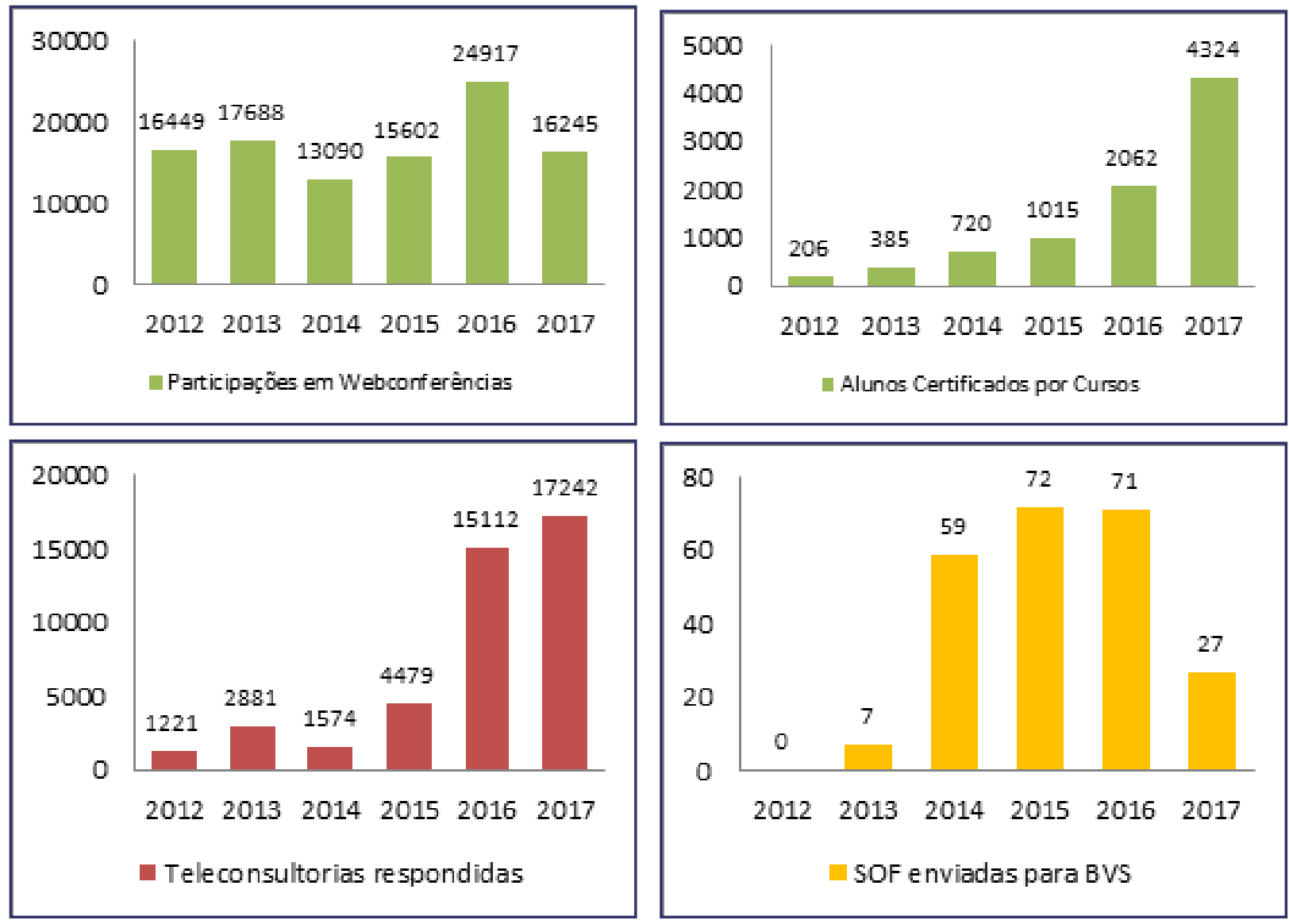


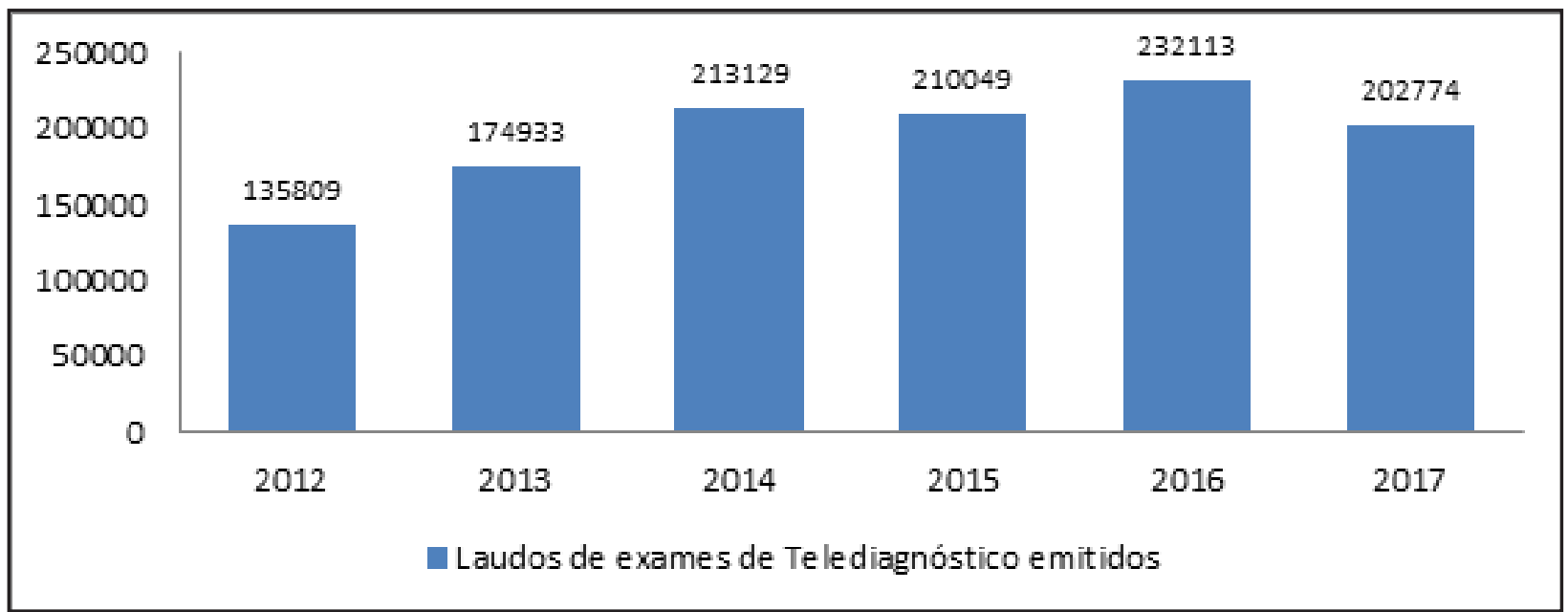

Fonte: Núcleo Telessaúde Santa Catarina, 2017a.

\section{TELESSAÚDE COMO TECNOLOGIA SOCIAL}

No contexto do SUS, consideram-se tecnologias em saúde: medicamentos, materiais, equipamentos e procedimentos, sistemas organizacionais, educacionais, de informações e de suporte, e programas e protocolos assistenciais que orientam a atenção e os cuidados à saúde das pessoas (BRASIL, 2005). Conceitualmente, o termo 'tecnologias em saúde' aponta para as ferramentas necessárias à ação transformadora, o que envolve procedimentos e o saber (SCHRAIBER; MOTA; NOVAES, 2015).

Até o final do século XX as tecnologias em saúde eram tratadas quase como sinônimo de tecnologias na medicina, quando passaram a ser pensadas de forma mais ampla, integrando a relação produto-processo e as relações sociais em que estão inseridos os atores que a constroem. Neste contexto, o emprego da tecnologia em saúde desdobra-se em duas concepções: a do saber tecnológico para a produção dos cuidados em saúde, e a dos modelos tecnológicos do trabalho, que trata da organização para um modo de produzir. A partir da organização para a produção, pode-se falar de modelos tecno-assistenciais ou modelos assistenciais em saúde (SCHRAIBER; MOTA; NOVAES, 2015).

A saúde pública exige a ampliação da conceituação de tecnologia para possibilitar uma construção teórica que responda à complexidade de exigências e necessidades que percorrem suas práticas. Para que essas práticas sejam discutidas em sentido tecnológico, exigem a configuração de arranjos concretos e materiais de trabalho, e tal trabalho abrange a integração de ações e saberes e a distinção entre a complexidade da tecnologia material que dispõe e da complexa tecnologia assistencial que requer para a construção dos cuidados (SCHRAIBER; MOTA; NOVAES, 2015).

Mehry (2002) apresenta uma conceituação para tecnologia que inclui os saberes que são utilizados para a produção do cuidado em saúde e que envolve a relação do profissional com os recursos técnicos que o servem e com os pacientes. Ele classifica tecnologia em três tipos: duras, leve-duras e leves, identificando cada uma delas como caixas de ferramentas. As tecnologias duras são a primeira caixa de ferramentas e compreendem equipamentos e máquinas que produzem material estruturado, como exames, medicamentos e outros recursos que servem à prática 
terapêutica. As tecnologias leve-duras compreendem à caixa que oferece as ferramentas que tornam possível significar como atos de saúde as tecnologias duras. Leve-duras são, portanto, as tecnologias referentes ao saber que orienta o trabalho em saúde e a relação do profissional de saúde com seus pacientes, a partir de conhecimento previamente estruturado - protocolos, evidências clínicas, epidemiologia, etc. -, que pode ser conduzido de forma dura e engessada, ou leve e maleável, o que é determinado pela forma como se dá a relação profissional-paciente (MEHRY, 2002; MEHRY; FEUERWARKER, 200?; DA ROS; MAEYAMA; LEOPARDI, 2012). E, por fim, as tecnologias leves estão na caixa das ferramentas subjetivas, que são produzidas nas relações e nas interações entre profissionais e pacientes, a partir da escuta, e envolvem a formação de vínculo, o acolhimento, a confiança, as singularidades; em uma troca que enriquece a ambos e permite ao profissional ampliar as suas caixinhas e aprender também com seus pacientes. Aqui têm importância as relações, o respeito e a ética, e há capacidade de os atores serem 'afetados' pelas trocas entre si (MEHRY, 2002; MEHRY; FEUERWARKER, 200?).

Pode-se pensar telessaúde no contexto do SUS como tecnologias duras e leve-duras que servem às tecnologias leves. A telessaúde como tecnologia dura contempla a necessidade de uma infraestrutura tecnológica - computadores, internet, equipamentos de som e imagem -, por meio da qual os profissionais poderão acessar os serviços. Já telessaúde como tecnologia levedura pode ser pensada como a oferta de apoio por meio de serviços alicerçados em conhecimento preconcebido, baseado em evidências científicas, e que é utilizado para que sejam respondidas as teleconsultorias, laudados os exames, oferecidos cursos, palestras ou outros espaços formadores (DA ROS; MAEYAMA; LEOPARDI, 2012).

O apoio ofertado por meio dos serviços de telessaúde promove a construção de conhecimento e qualificação dos profissionais de saúde, por meio dos quais se fortalece a APS e se amplia o seu potencial de promoção de inclusão e justiça social a partir de respostas positivas às necessidades dos usuários que procuram os serviços de saúde, nesse sentido, a promoção das tecnologias leves.

Quando se fala dessa qualificação das práticas sanitárias, está-se tratando de tecnologia social. O apoio oferecido pela telessaúde promove fortalecimento da APS por meio de qualificação dos profissionais, e permite a transformação da realidade com foco nos usuários do sistema.

Profissionais amparados e mais seguros para olhar para os pacientes de forma mais acolhedora e oferecer respostas positivas às suas necessidades de saúde. Um exemplo claro disso é a facilitação e a ampliação do acesso à assistência, promovida diretamente pelos serviços de teleconsultoria e telediagnóstico e pelo Telessaúde de forma geral.

Com esse trabalho, o Telessaúde SC tem contribuído com a busca pelo modelo de atenção integral proposto pelo SUS, que se orienta por uma APS forte, com o objetivo de melhorar o cuidado por meio do trabalho de equipes de saúde da família preparadas e qualificadas (MALTA et al., 2016).

\section{CONSIDERAÇÕES FINAIS}

O Núcleo Telessaúde SC busca a integração entre o saber acadêmico e do serviço, fomentando a transformação do modelo de atenção hegemonicamente biológico, para o alcance de um modelo integral e que responda às necessidades de saúde da população, uma vez que o 
Telessaúde utiliza o referencial da APS; e a teleconsultoria, a tele-educação e a segunda opinião formativa atuam tanto na dimensão clínica quanto na organização do processo de trabalho, à exceção do telediagnóstico, no qual o enfoque é eminentemente clínico.

Para tanto, há um esforço em cumprir os objetivos do Telessaúde Brasil Redes adaptados à realidade e às necessidades locais, mantendo sempre o contato com os profissionais e os trabalhadores de saúde para construir serviços úteis.

Esse processo se fortalece na medida em que todos os profissionais que compõem as equipes multiprofissionais são ouvidos e podem utilizar os serviços de acordo com interesses individuais ou coletivos, indicar fragilidades e fortalecer potencialidades em parceria com seus colegas e com a equipe do Telessaúde SC.

Apesar de expressiva, a apresentação exclusivamente de dados quantitativos do uso dos serviços do Telessaúde SC não permite conclusões acerca do alcance e da qualidade desse acesso e aplicação pelos profissionais. Nesse sentido, é preciso que sejam pensados trabalhos que permitam aprofundar o conhecimento e avaliar a oferta e a utilização dos serviços de telessaúde, de forma que sejam minimizadas as fragilidades e maximizadas as potencialidades para o alcance dos seus objetivos.

\section{AGRADECIMENTOS}

Ao Núcleo Telessaúde Santa Catarina, a toda a sua equipe e aos profissionais de saúde, gestores e parceiros que fazem todo esse trabalho ter sentido.

À CAPES, pela bolsa de estudos durante o desenvolvimento deste trabalho.

\section{REFERÊNCIAS}

ALKMIM, M. B. M. et al. Hospital das Clínicas da Universidade Federal de Minas Gerais (UFMG). In: MESSINA, L. A., RIBEIRO FILHO, J. L. Impactos da rede universitária de telemedicina: ações de educação contínua, pesquisa colaborativa e assistência remota: Fase I (2006-2009). Rio de Janeiro: E-papers, 2013. p.39-46.

AMERICAN TELEMEDICINE ASSOCIATION. What is Telemedicine? 2016. Disponível em: < http:// www.americantelemed.org/about/about-telemedicine>. Acesso em: 14 fev. 2016.

ASCENCIO, A. C. S. A Teleducação interativa na capacitação de profissionais em saúde auditiva. 2012. 135f. Dissertação (Mestrado em Fonoaudiologia) - Universidade de São Paulo, Bauru, 2012.

BILL, G.; CRISCI, C. D.; CANET, T. La Red de Telesalud de las Américas y su papel em la atención primaria de la salud. Revista Panamericana de Salud Publica, v. 35, n. 5/6, p.442-445, 2014.

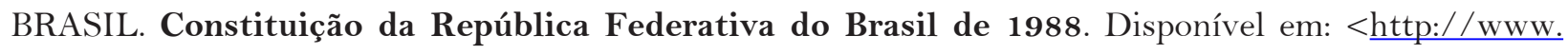
planalto.gov.br/ccivil 03/constituicao/ConstituicaoCompilado.htm>. Acesso em: 15 fev. 2016.

BRASIL. Ministério da Saúde. Lei $\mathbf{n}^{\mathbf{0}}$ 8.080, de 19 de setembro de 1990: Dispõe sobre as condições para a promoção, proteção e recuperação da saúde, a organização e o funcionamento dos serviços correspondentes e dá outras providências. Disponível em: < $\underline{\text { http://www.planalto.gov.br/ccivil 03/Leis/ }}$

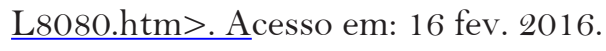

BRASIL. Ministério da Saúde. Manual de Telessaúde para Atenção Primária à Saúde. Disponível em: <http://189.28.128.100/dab/docs/portaldab/publicacoes/manual_telessaude.pdf >. Acesso em: 12 mar. 2017. 
BRASIL. Ministério da Saúde. Política Nacional de Educação Permanente em Saúde. Disponível em: $<$ http://bvsms.saude.gov.br/bvs/publicacoes/politica_nacional_educacao Acesso em: 15 fev. 2016.

BRASIL. Ministério da Saúde. Portaria no 2.5 10, de 19 de dezembro de 2005. Disponível em: < $\underline{\text { http:// }}$ www.funasa.gov.br/site/wp-content/files_mf/Pm 2510_2005.pdf >. Acesso 14 dezembro 2015.

BRASIL. Ministério da Saúde. Portaria n 2.546, de 27 de outubro de 2011: redefine e amplia o Programa Telessaúde Brasil, que passa a ser denominado Programa Nacional Telessaúde Brasil Redes.

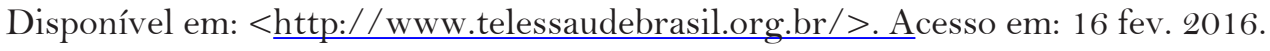

BRASIL. Ministério da Saúde. Portaria $\mathbf{n}^{\mathbf{0}}$ 2.554, de 28 de outubro de 2011 : institui, no Programa de Requalificação de Unidades Básicas de Saúde, o Componente de Informatização e Telessaúde Brasil Redes na Atenção Primária, integrado ao Programa Nacional Telessaúde Brasil Redes. Disponível em: < $\underline{\text { http:// }}$ www.telessaudebrasil.org.br/>. Acesso em: 16 fev. 2016.

BRASIL. Ministério da Saúde. Portaria no 35, de 4 de janeiro de 2007: Institui, no âmbito do Ministério da Saúde, o Programa Nacional de Telessaúde. Diário Oficial da União, 05 de janeiro de 2007.

BRASIL. Ministério da Saúde. Núcleos de Telessaúde. Disponível em: <http://dab.saude.gov.br/ portaldab/ape_telessaude.php?conteudo=nucleos_telessaude $>$. Acesso em: 10 jun. 2016.

BRASIL. Ministério da Saúde. Custeio dos núcleos de telessaúde: manual instrutivo. Brasília: Ministério da Saúde, 2015a.

BRASIL. Ministério da Saúde. Nota Técnica no 50/2015-DEGES/SGTES/MS: Diretrizes para a oferta de atividades do Programa Nacional Telessaúde Brasil Redes. Brasília: Ministério da Saúde, $2015 \mathrm{~b}$. Disponível em: <http:// 189.28.128.100/dab/docs/portaldab/notas_tecnicas/Nota_Tecnica_Diretrizes_ Telessaude.pdf $\geq$. Acesso em: 08 jun. 2016.

CÁCERES MÉNDEZ, E.A. et al. Telemedicina: historia, aplicaciones y nuevas herramientas en el aprendizaje. Universitas Médica, Bogotá, v.52, n.1, p.11-35, 2011.

DA ROS, M. A.; MAEYAMA, M. A.; LEOPARDI, M. T. Tecnologia na área da saúde. De que tecnologia estamos falando? Saúde e Transformação Social, Florianópolis, v.3, n.3, p.29-35, 2012.

EL-KHOURI, S. G. Telemedicina: análise da sua evolução no Brasil. 2003. 247f. Dissertação (Mestrado em Medicina) - Universidade de São Paulo, São Paulo, 2003.

FIGUEIREDO, A. M.; GUEDES, T. A. L. Programa Nacional Telessaúde Brasil Redes. In: VALENTIM, R. A. M. et al. (Ed.). A Telessaúde no Brasil e a inovação tecnológica na atenção primária. Natal: EDUFRN, 2015. p.27-46.

MALTA, D. C. et al. A Cobertura da Estratégia de Saúde da Família (ESF) no Brasil, segundo a Pesquisa Nacional de Saúde, 2013. Ciência \& Saúde Coletiva, Rio de Janeiro, v.2 1, n.2, p.327-338, 2016.

MARCOLINO, M. S. et al. A Rede de Teleassistência de Minas Gerais e suas contribuições para atingir os princípios de universalidade, equidade e integralidade do SUS - relato de experiência. Revista Eletrônica de Comunicação, Informação e Inovação em Saúde, Rio de Janeiro, v.7, n.2, p.1-2 1, 2013.

MERHY, E. E. Saúde: a cartografia do trabalho vivo. São Paulo: Hucitec, 2002.

MERHY, E. E.; FEUERWERKER, L. C. M. Novo olhar sobre as tecnologias de saúde: uma necessidade contemporânea. Disponível em: <http://www.uff.br/saudecoletiva/professores/merhy/capitulos-25. pdf>. Acesso em: 07 nov. 2017. 
MONTEIRO, A. M. V. et al. Universidade do Estado do Rio de Janeiro (UERJ). In: MESSINA, L. A.; RIBEIRO FILHO, J. L. Impactos da rede universitária de telemedicina: ações de educação contínua, pesquisa colaborativa e assistência remota: Fase I (2006-2009). Rio de Janeiro: E-papers, 2013. p.67-71.

NASSIF, L. O programa brasileiro de telessaúde. Disponível em: < http://jornalggn.com.br/ noticia/ o-programa-brasileiro-de-telessaude>. Acesso em: 17 out. 2015.

NILSON, L. G. et al. Telehealth Centers: A Proposal of a Theoretical Model for Evaluation. Telemedicine and e-Health Mary Ann Liebert, Inc. v.23, n.11, p.905-912, nov. 2017.

NÚCLEO TELESSAÚDE SANTA CATARINA. Universidade Federal de Santa Catarina. Relatórios e planilhas de monitoramento dos serviços do Telessaúde SC referentes ao período de 2012 a outubro de 2017. Florianópolis: Universidade Federal de Santa Catarina. 2017 a.

NÚCLEO TELESSAÚDE SANTA CATARINA. Universidade Federal de Santa Catarina. Brochura do Núcleo Telessaúde SC. Florianópolis: Universidade Federal de Santa Catarina. 2017b.

OLIVIERA, D. G. et al. Análise da implantação do Programa Telessaúde Brasil em Pernambuco, Brasil: estudo de casos. Cadernos de Saúde Pública, Rio de Janeiro, v.31, n.11, p.2379-2389, nov. 2015.

ORGANIZAÇÃO MUNDIAL DA SAÚDE. Capacidades Humanas para a Saúde Telessaúde. Disponível em: <http://www.paho.org/bra/index.php?option=com_ content\&view=article\&id=256\&It emid=373>. Acesso em: 28 fev. 2016.

ORGANIZAÇÃO PANAMERICANA DE LA SALUD. Marco de Implementación de um Servicio de Telemedicina. Disponível em: <http://iris.paho.org/xmlui/bitstream/handle/ 123456789/28413/9789275319031_spa.pdf? sequence=5\&isAllowed=y $\geq$. Acesso em: 14 jun. 2016.

PAIM, J. et al. The Brazilian health system: history, advances, and challenges. The lancet. v.377, n. 9779, p.1778-1797, maio 2011.

PINTO, H. A. et al. Atenção Primária e Educação Permanente em Saúde: cenário apontado pelo Programa Nacional de Melhoria do Acesso e da Qualidade da Atenção Primária (PMAQ-AB). Divulgação em Saúde para Debate, Rio de Janeiro, n.5 1, p.145-60, out. 2014.

SAVARIS, A. et al. O uso da telemedicina assíncrona em larga escala no setor público de saúde. Disponível em: <https://www.researchgate.net/profile/Douglas_Macedo/publication/228437024_O_ Uso_da_Telemedicina_Assistencial_Assincrona_em_Larga_Escala_no_Setor_Publico_de_Saude/ links/02e7e52faod8d506bdo00000/O-Uso-da-Telemedicina-Assistencial-Assincrona-em-Larga-Escalano-Setor-Publico-de-Saude.pdf $\geq$. Acesso em: 18 jun. 2016.

SCHRAIBER, L. B.; MOTA, A.; NOVAES, H. M. D. Tecnologias em saúde. Disponível em: < $\underline{\text { http:// }}$ www.sites.epsjv.fiocruz.br/dicionario/verbetes/tecsau.html\#topo $>$. Acesso em: 04 out. 2015.

SILVA, A. B. Telessaúde no Brasil - conceitos e aplicações. Rio de Janeiro: DOC, 2014.

SILVA, K. C. L. Análise do Programa Telessaúde Brasil Redes no estado de Pernambuco no período de 2007 a 2011 . 2013. 162f. Dissertação (Mestrado em Saúde Pública) - Fundação Oswaldo Cruz, Recife, 2013.

SIMÕES, N. et al. A Rede Universitária de Telemedicina - RUTE. In: MESSINA, L. A.; RIBEIRO FILHO, J. L. Impactos da rede universitária de telemedicina: ações de educação contínua, pesquisa colaborativa e assistência remota: Fase I (2006-2009). Rio de Janeiro: E-papers, 2013. p.11-36. 
TELESSAÚdE BRASIL REDES. História do Telessaúde. Disponível em: <http://www. telessaudebrasil.org.br>. Acesso em: 16 fev. 2016.

VON WANGEHEIM, A.; CAVALCANTE, C.; WAGNER, H. Hospital Universitário da Universidade Federal de Santa Catarina (UFSC): Telemedicina e Telessaúde. In: MESSINA, L. A.; RIBEIRO FILHO, J. L. Impactos da rede universitária de telemedicina: ações de educação contínua, pesquisa colaborativa e assistência remota: Fase I (2006-2009). Rio de Janeiro: E-papers, 2013. p.158-171.

WEN, C. L. Faculdade de Medicina da Universidade de São Paulo (FMUSP): A Telemedicina e a Educação Interativa. In: MESSINA, L. A.; RIBEIRO FILHO, J. L. Impactos da rede universitária de telemedicina: ações de educação contínua, pesquisa colaborativa e assistência remota: Fase I (2006-2009). Rio de Janeiro: E-papers, 2013. p.55-66.

WEN, C.L. Telemedicina e Telessaúde: Um panorama no Brasil. Informática Pública, ano 10, n. 2, p. $7-15,2008$. 